\title{
Fast ferroelectric phase shifters for energy recovery linacs
}

\author{
S. Yu Kazakov, ${ }^{1,2}$ S. V. Shchelkunov, ${ }^{3, *}$ V. P. Yakovlev, ${ }^{2}$ A. Kanareykin, ${ }^{4}$ E. Nenasheva, ${ }^{5}$ and J. L. Hirshfield ${ }^{1,3}$ \\ ${ }^{1}$ Omega-P, Inc., 258 Bradley Street, New Haven, Connecticut 06510, USA \\ ${ }^{2}$ Fermi National Accelerator Laboratory, Batavia, Illinois 60510, USA \\ ${ }^{3}$ Beam Physics Laboratory, Yale University, 272 Whitney Avenue, New Haven, Connecticut 06511, USA \\ ${ }^{4}$ Euclid Techlabs LLC, Solon, Ohio 44139, USA \\ ${ }^{5}$ Ceramics Ltd., St. Petersburg, 194223, Russia \\ (Received 18 May 2010; published 24 November 2010)
}

\begin{abstract}
Fast phase shifters are described that use a novel barium strontium titanate ceramic that can rapidly change its dielectric constant as an external bias voltage is changed. These phase shifters promise to reduce by $\sim 10$ times the power requirements for the rf source needed to drive an energy recovery linac (ERL). Such phase shifters will be coupled with superconducting radiofrequency cavities so as to tune them to compensate for phase instabilities, whether beam-driven or those caused by microphonics. The most promising design is presented, which was successfully cold tested and demonstrated a switching speed of $\sim 30 \mathrm{~ns}$ for $77 \mathrm{deg}$, corresponding to $<0.5 \mathrm{~ns}$ per deg of rf phase. Other crucial issues (losses, phase shift values, etc.) are discussed.
\end{abstract}

DOI: 10.1103/PhysRevSTAB.13.113501

PACS numbers: $84.40 .-\mathrm{x}, 41.90 .+\mathrm{e}, 29.20 .-\mathrm{c}$

\section{INTRODUCTION}

In energy recovery linacs (ERLs) there are several factors which significantly affect the required wall-plug power. With small beam loading, rf power requirements are determined by Ohmic wall losses, imbalance between beam currents, and microphonics. Compensation for the latter two typically requires a rapid change in coupling between the cavity and feeding line, and attendant high bandwidth, leading to a need for significant additional rf power. If beam loading is not small, there are beam-driven phase instabilities for which compensation will also demand additional power.

Compensation can be either by changing the cavity geometry to offset detuning caused by phase instabilities and/or microphonics [1,2], and/or to apply a corrective phase shift to the reflected rf wave that is reintroduced to the cavity so as to cancel phase instabilities $[3,4]$. The first strategy is accomplished by internal or external motors, or fast internal mechanical piezoelectric tuners. The second approach utilizes fast ferrite or ferroelectric phase shifters that are external to the cryomodules, whereas piezoelectric and other mechanical tuners require operation at cryogenic temperatures and thus permit only limited access in the event of a failure. Further, piezoelectric devices have mechanical resonances which may interfere with control system performance if their own resonance frequency overlaps with the microphonics excitation to be controlled [5]. It has been reported that the piezoelectric tuners may operate as fast as $\sim 25 \mathrm{kHz}$ ([see, e.g., Ref. [6], slide 11); however, it is still unknown if piezoelectric tuners are efficient enough at high frequencies.

\footnotetext{
*sergey.shchelkunov@gmail.com
}

Ferrite phase shifters [7-9] are presently limited in their response time to $\sim 30 \mu \mathrm{s}$, while the required response time may be only a few $\mu$ s. The limitation comes mainly from the eddy currents in the ferrite material [8].

The need for $\mu$ s response time is dictated by the phase and amplitude stability requirements of $\sim 0.06 \mathrm{deg}$ and $3 \times 10^{-4}$, as cited for the Cornell ERL [10]; requirements are similar for the electron cooler project at BNL [11]. The gain in the control feedback loop should be high enough, and its bandwidth wide enough, to ensure this high degree of stability. This translates to a bandwidth of about $1 \mathrm{MHz}$, and rules out contemporary ferrite tuners.

The authors have studied several designs for a fast electrically controlled ferroelectric phase shifter for ERL applications. The device is to allow changing the rf coupling during the cavity filling process in order to effect significant power savings, and also to provide rapid compensation for beam imbalance and allow for fast stabilization against phase fluctuations caused by microphonics and beam-driven instabilities. This capability should allow a reduction by about an order of magnitude in the required power from the rf source.

\section{POSSIBLE RF-POWER SAVINGS}

The rf power $P_{g}$ required to maintain an accelerating voltage $V$ is given by [12]

$$
\begin{aligned}
P_{g}= & \frac{V^{2}(1+\beta)^{2}}{4 \beta Q_{0}(r / Q)}\left\{\left(1+\frac{I_{\mathrm{Re}}(r / Q) Q_{0}}{V(1+\beta)}\right)^{2}\right. \\
& \left.+\left[\frac{Q_{0}}{1+\beta}\left(\frac{\omega_{0}}{\omega}-\frac{\omega}{\omega_{0}}\right)-\frac{I_{\mathrm{Im}}(r / Q) Q_{0}}{V(1+\beta)}\right]^{2}\right\},
\end{aligned}
$$

where $\omega_{0}$ is the cavity resonance frequency; $Q_{0}$ is its unloaded quality factor; $\beta$ is the coupling factor, for SC 
cavity $\beta \gg 1 ; r / Q$ is the cavity impedance; $I_{\mathrm{Re}}=$ $I\left(\cos \delta \phi_{a}-\cos \delta \phi_{d}\right), \quad I_{\mathrm{Im}}=I\left(\sin \delta \phi_{a}-\sin \delta \phi_{d}\right), \quad \delta \phi_{a}$ and $\delta \phi_{d}$, are the average phases of the accelerating and decelerating beams compared with the rf phase; and $I$ is the beam current. The value $\delta \omega=\omega_{0}-\omega$ is determined by the amplitude of uncontrolled noise.

In $[11,13]$, an example is given for a cooler linac having two cavities with $Q_{0} \approx 4.5 \times 10^{10}$ at $2 \mathrm{~K}$ and $r / Q \approx$ $400 \mathrm{Ohms} /$ cavity, $I=50 \mathrm{~mA} \times 2=100 \mathrm{~mA}$ and $V \approx$ $25 \mathrm{MV}$. (The beams go through the linac twice.) The intrinsic rf power required for the parameters listed above,

$$
P_{\mathrm{int}}=\frac{V^{2}}{Q_{0}(r / Q)}=9 \mathrm{~W}
$$

If the accelerated and decelerated beams are well balanced, and the beams are in phase with the rf field, the required power is determined by the peak frequency variations caused by microphonics [2], namely,

$$
P_{g}=\frac{V^{2}}{4 Q_{l}(r / Q)}\left[1+\left(2 Q_{l} \frac{\delta \omega}{\omega}\right)^{2}\right]
$$

where $Q_{l}$ is loaded quality factor, $Q_{l}=Q_{0} /(1+\beta)$. One finds the optimal value of the loaded quality factor, $Q_{\mathrm{opt}}=$ $\omega / 2 \delta \omega$, and the minimum required power is proportional the peak cavity detuning, namely,

$$
P_{g}=\frac{V^{2}}{(r / Q)} \frac{\delta \omega}{\omega}=0.55 \mathrm{~kW} \times d f[\mathrm{~Hz}]
$$

where $\delta f=\delta \omega / 2 \pi$ is the peak microphonic cavity detuning in Hz. If, for example, the peak cavity detuning is reduced to $30 \mathrm{~Hz}$ (a typical value), the required input power would be $\sim 17 \mathrm{~kW}$ for four five-cell cavities.

While beam loss within reasonable limits gives no significant increase in required power, the phase error $\delta \phi$ of the beams does, because in this case the beam introduces an additional reactance proportional to $\delta \phi$, as can be seen from (1). The required power in this case is

$$
\begin{aligned}
P_{g} & =\frac{V^{2}}{(r / Q)}\left(\frac{\delta \omega}{\omega}+\frac{(r / Q) I \delta \varphi}{2 V}\right) \\
& =0.55 \mathrm{~kW} \times d f[\mathrm{~Hz}]+22 \mathrm{~kW} \times \delta \varphi\left[^{\circ}\right] .
\end{aligned}
$$

With, for example, $\delta \phi=1^{\circ}$ and $\delta f=30 \mathrm{~Hz}$, the required power would be about $40 \mathrm{~kW}$. Obviously, it is crucial to provide a means for compensation of phase instabilities in the cited example, as well as in any other ERL [see, e.g., [14], slides 42-44] to keep the rf-power requirements to a minimum.

The most popular scheme [7-9] among several others to utilize phase shifters is shown in Fig. 1

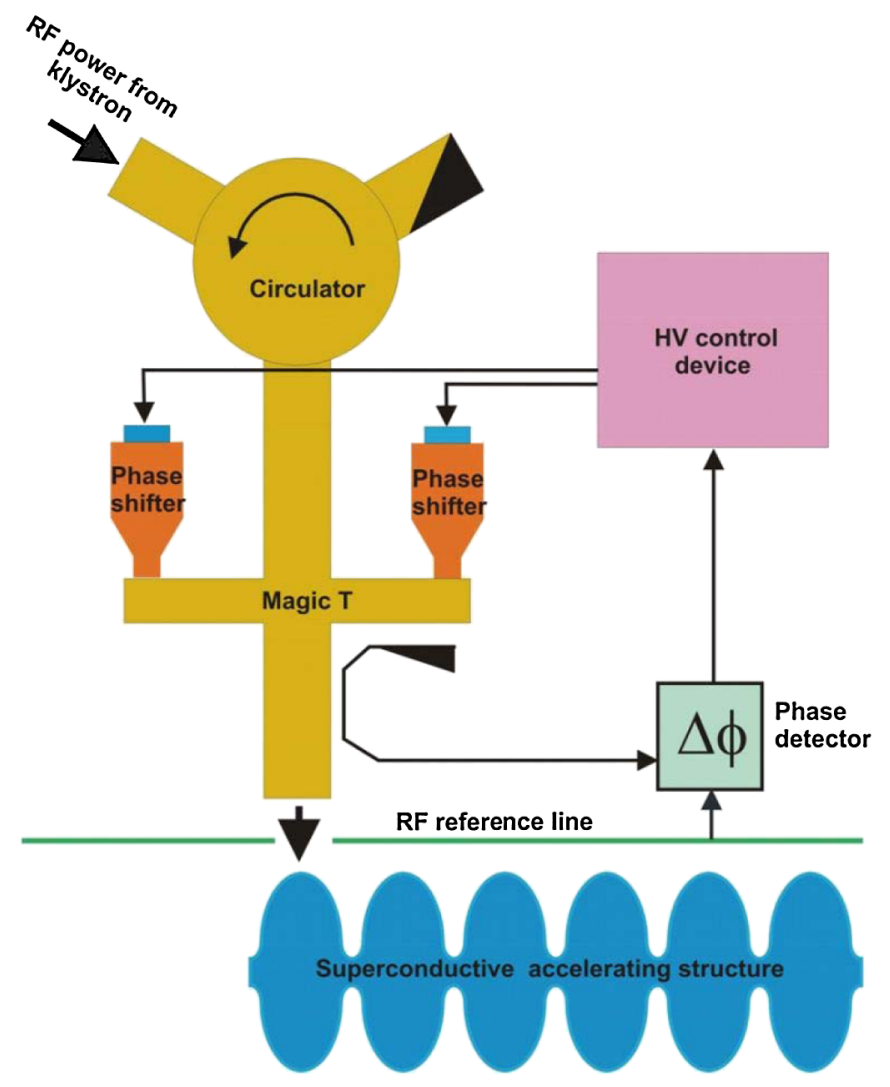

FIG. 1. External tuner in a magic- $T$ configuration with adjustable phase shifters. Each phase shifter works in one-port configuration, with its second port being shortened.

\section{FERROELECTRIC MATERIAL AS BASIS OF THE PROPOSED DEVICE}

Recently, ferroelectric (FE) devices for fast switching applications have received close attention, and are already used up to $100 \mathrm{~kW}$ peak in military systems [15], phasedarray radars [16], and communication systems [17]. FE's have a dielectric permittivity $\varepsilon(\mathbf{E})$ that depends on electric field $\mathbf{E}$, and can be rapidly altered by application of an external bias-voltage pulse. The response time would be limited by that of the external bias circuit. The minimum intrinsic switching time demonstrated is less than $1 \mathrm{~ns}$ [17]. Modern bulk ferroelectrics, e.g. $\mathrm{Ba}_{x} \mathrm{Sr}_{1-x} \mathrm{TiO}_{3}$ (barium strontium titanate or BST) with $\varepsilon \sim 500$, have sufficiently high electric-breakdown strength $(100-200 \mathrm{kV} / \mathrm{cm})$ and require an acceptable bias electric field $(\sim 20-50 \mathrm{kV} / \mathrm{cm})$ to effect a $20 \%-30 \%$ change in $\varepsilon$. Loss tangent for commercially available samples is about $\sim 1.5 \times 10^{-3}$ at $1 \mathrm{GHz}$ [16].

Euclid Techlabs LLC recently developed and tested a modified bulk FE [18] based on a composition of BST ceramics, magnesium compounds, and rare-earth metal oxides. The availability of this FE already allows one to create a high-power rf phase shifter with the peak power required for ERL. 
TABLE I. Properties of modified BST ferroelectric ceramic.

\begin{tabular}{|c|c|}
\hline Dielectric constant, $\varepsilon$ & $\sim 460$ \\
\hline Tunability, $\partial \varepsilon / \partial E_{\text {bias }}$ & $>2 /(\mathrm{kV} / \mathrm{cm})$ \\
\hline Intrinsic response time & $<10$ ns \\
\hline Loss tangent at $1.3-1.4 \mathrm{GHz}, \tan (\delta)$ & $2 \times 10^{-3}$ \\
\hline Loss tangent at $700-900 \mathrm{MHz}, \tan (\delta)$ & $1.1 \times 10^{-3}$ \\
\hline Breakdown limit & $200 \mathrm{kV} / \mathrm{cm}$ \\
\hline Thermal conductivity, $K$ & $7.02 \mathrm{~W} / \mathrm{m} \mathrm{K}$ \\
\hline Specific heat, $C$ & $0.605 \mathrm{~kJ} / \mathrm{kg} \mathrm{K}$ \\
\hline Density, $\rho$ & $4.86 \mathrm{~g} / \mathrm{cm}^{3}$ \\
\hline Coefficient of thermal expansion & $10.1 \times 10^{-6} \mathrm{~K}^{-1}$ \\
\hline Temperature tolerance, $\partial \varepsilon / \partial T$ & $3 \mathrm{~K}^{-1}$ \\
\hline
\end{tabular}

Properties of modified BST FE ceramic are in Table I.

For the proposed devices, the FE ceramic is manufactured in the form of rings [Fig. 2(a)] or bars [Fig. 2(b)]. To measure the loss tangent for ringlike samples, the setup

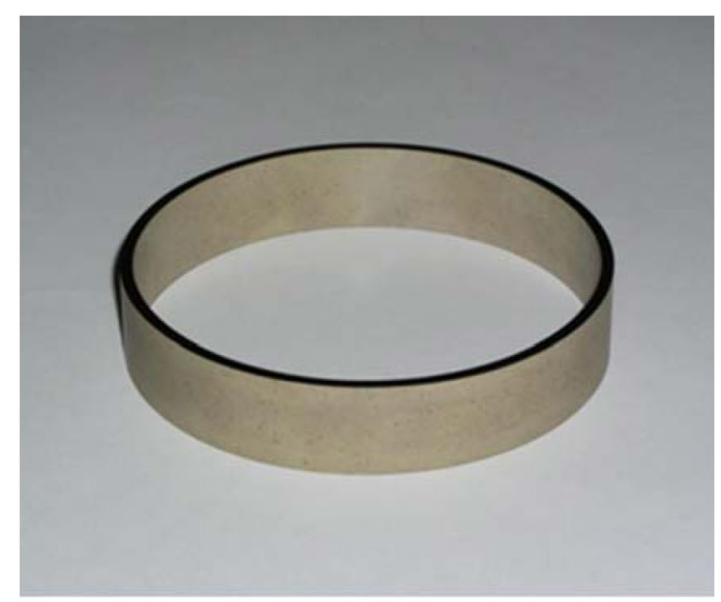

(a)

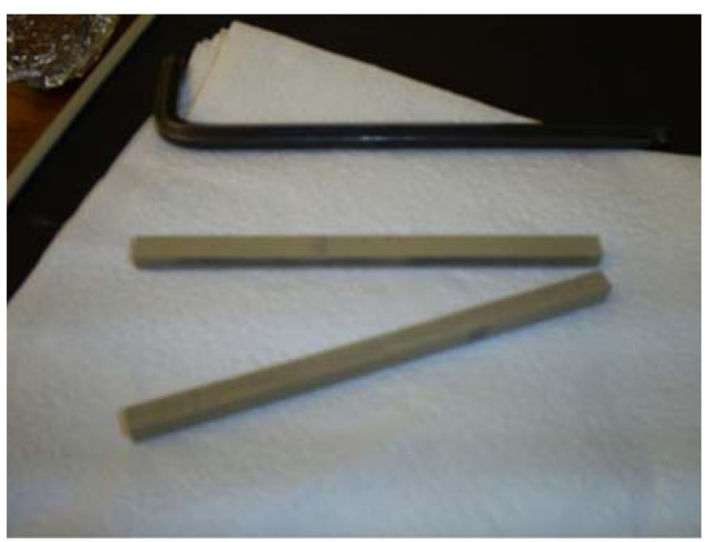

(b)

FIG. 2. (a) FE ceramic ring $\varnothing 106 \times 2.8 \times 22 \mathrm{~mm}$. Typically the top and bottom of the ring are fully coated with a thin, goldbased metallization layer (in a process proprietary to Ceramic, Ltd. (St. Petersburg, 194223, Russia). (b) FE ceramic bars $(6 \times 5 \times 108 \mathrm{~mm})$. shown in Fig. 3 was used [19]. Measurements on the bars were done with the bars suspended along the axis in a long metal pipe. In both cases, the values of the loss tangent were inferred in a manner as described in the caption to Fig. 3

Figure 4 presents results of measurements of tunability [changes (or relative changes) in the dielectric constant when an external electric field or voltage is applied]. To measure the tunability, one for instance uses a setup similar to that in Fig. 3, and applies a bias to upper and lower halves of the cavity thereby creating an electric field across the ring(s). The changes in the mode spectrum are observed, and the frequencies with and without the applied electric field are compared. Again, using e.g. HFSS, one forces the simulated modes' frequencies to undergo the same change

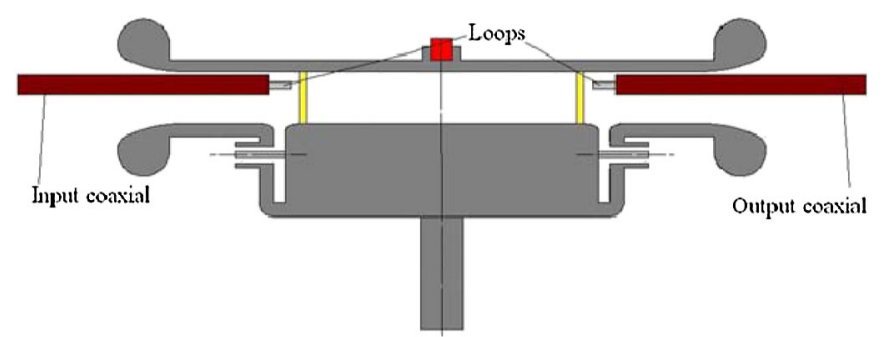

FIG. 3. An example of setup to measure FE ring loss tangent. Because of a large dielectric constant of the ceramic, modes exist with their fields almost entirely contained in the ceramic. In measurements, one may excite these modes using loops, measure their $Q$ factors, and then in, e.g., HFSS simulations force the simulated $Q$ factors be as the observed ones by adjusting the loss tangent of the ceramic in the simulation model which reproduces the real configuration. Hence, the value of the loss tangent is inferred for a set of frequencies corresponding to the mode spectrum.
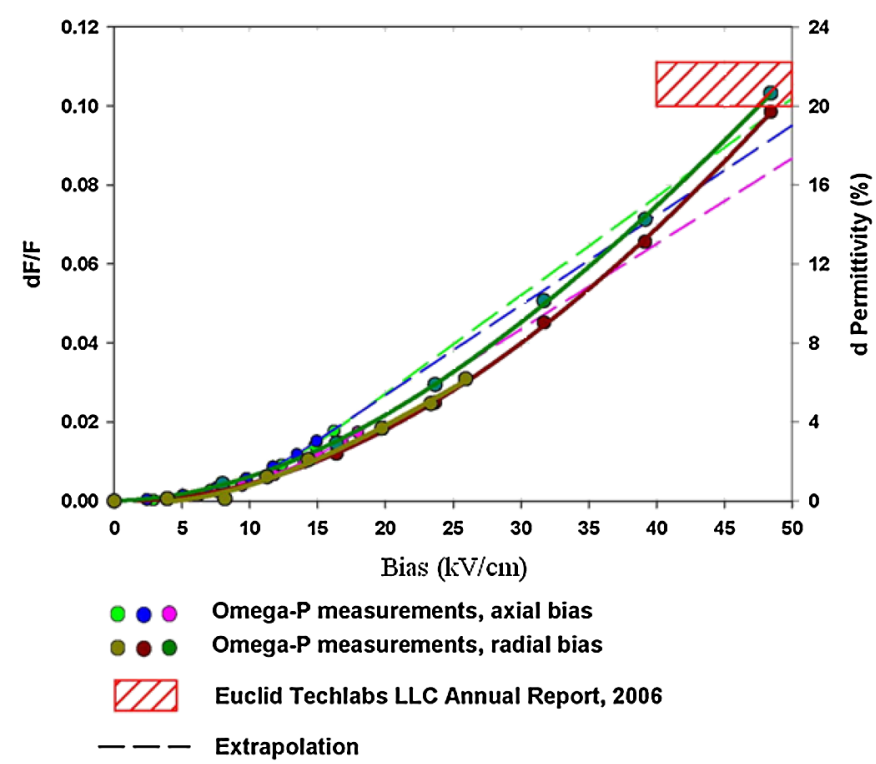

FIG. 4. Tunability measurements on modified BST ring. Note that one division on the right vertical axis is $4 \%$. 
by adjusting the dielectric constant of the ferroelectric material in the simulation model that reproduces the real configuration. Hence, the change in dielectric constant (and consequently, the tunability) can be inferred.

In Fig. 4, the lower portion of the curve indicates low tunability at lower applied voltages. Presently, efforts are underway to reduce the loss tangent at $1.3 \mathrm{GHz}$, without undue sacrifice of tunability.

\section{PHASE SHIFTER DESIGN}

Three configurations have been considered: coaxial, planar/coaxial hybrid, and sandwich-in-waveguide; all for $500 \mathrm{~kW}$ pulse and $4-5 \mathrm{~kW}$ average powers, figures dictated by ILC parameters that we have chosen as the base line. Below we describe the last of these which was successfully built, and cold tested.

The sandwich-in-waveguide configuration employs a standard WR650 waveguide as a host for three sets of two narrow ferroelectric (FE) bars and two matching ceramic slabs $(\varepsilon \sim 21)$, as shown in Fig. 5(a). Each set rests on a metal plate, with a second metal plate above, as seen in Fig. 5(b). The sides of the FE bars and matching slabs that are in contact with the metal electrodes are metalized with gold-based coating layers in a process that is proprietary to Ceramics, Ltd. (St. Petersburg, 194223, Russia).

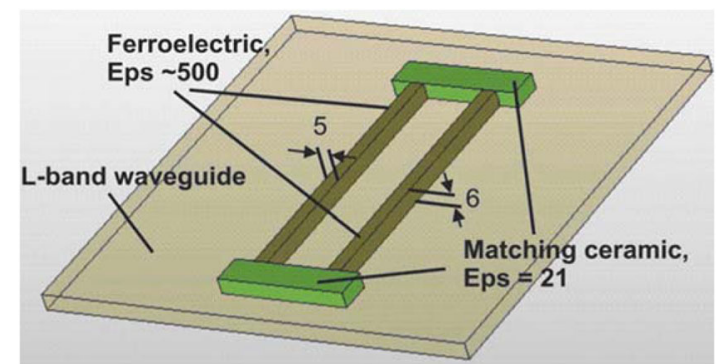

(a)

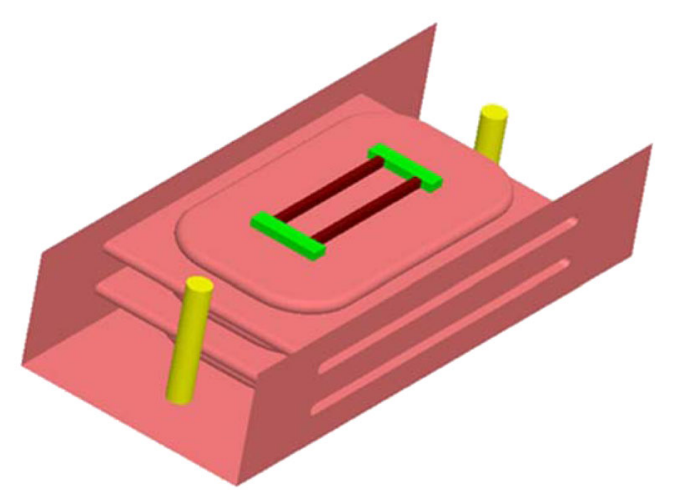

(b)

FIG. 5. (a) Arrangement for one set of FE bars (dark green) and ceramic slabs (light green); dimensions are in $\mathrm{mm}$. (b) WR650 waveguide with top removed to show three "sandwiches" and matching rods.

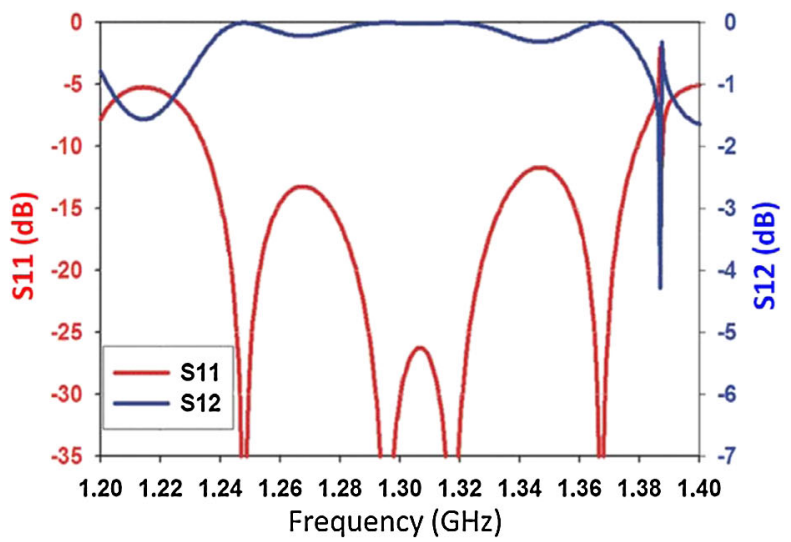

(a)

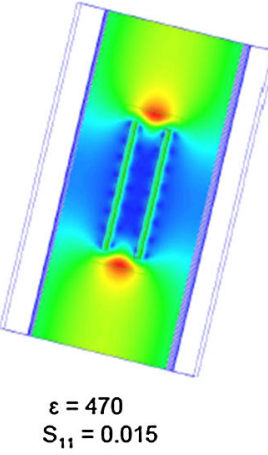

$S_{11}=0.015$

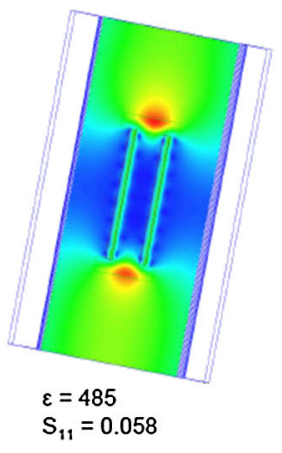

(b)

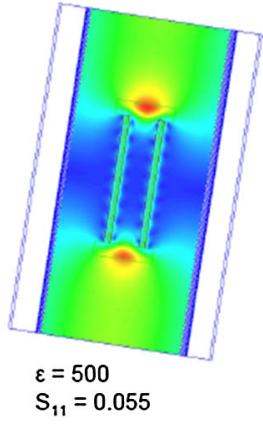

$\varepsilon=500$
FIG. 6. (a) Frequency response for sandwich geometry with $\varepsilon \sim 470$; it is nearly the same for $470<\varepsilon<500$. (b) Field pattern demonstrates that the geometry is well matched for different values of the dielectric constant of the ferroelectric.

Alternate plates are joined to an electric feedthrough to provide the desired bias, while other plates are grounded. When assembled, dimensions are $8.2 \mathrm{~cm} \times 16.5 \mathrm{~cm} \times$ $30 \mathrm{~cm}$. The mode spectrum is sparse, and can be controlled by changing the geometry. For matching to the structure, dielectric rods (alumina with $\varepsilon \sim 9.8$ ) are placed before and after the sandwiches. Frequency response is shown in Fig. 6(a).

Table II lists design parameters of the phase shifter for $500 \mathrm{~kW}$ of pulsed and $4 \mathrm{~kW}$ of average power.

\section{LOSS, PHASE SHIFT, AND SWITCHING SPEED MEASUREMENTS}

Low-power rf measurements were made using only one of the three sandwiches in a waveguide that has the same width as WR650, but tapered to one-third the standard height, as shown in Figs. 7(a) and 7(b). The central electrode can be biased electrically.

The loss tangent of ferroelectric bars is measured for the uncoated bars (manufactured from the same batch used to make the bars coated with gold-based metallization layers, as used in the $1 / 3$ model). The value of loss tangent is determined to be $\sim 2 \times 10^{-3}$, suggesting that the $1 / 3$ 
TABLE II. Design parameters of the phase shifter.

\begin{tabular}{lc}
\hline \hline FE permittivity $\varepsilon$ at $V_{\text {bias }}=0$, & 460 \\
$\partial$ (phase) $/ \partial \varepsilon$, deg & 4 \\
Maximum DC electric field, $\mathrm{kV} / \mathrm{cm}$ giving $\Delta$ (phase) $=120$ deg & 15 \\
Total loss, $\%$ & $2.8+6 \times 10^{3} \tan (\delta)$ \\
Maximum $E$ field in FE, $\mathrm{kV} / \mathrm{cm}$ & 3 \\
Maximum $E$ field in ceramic, $\mathrm{kV} / \mathrm{cm}$ & 5.9 \\
Maximum $E$ field in air, $\mathrm{kV} / \mathrm{cm}$ & 6.1 \\
Phase shift, deg, at $15 \mathrm{kV} / \mathrm{cm}$ bias & 120 \\
FE pulse heating with loss tan $5 \times 10^{-4}$ & $0.2 \mathrm{~K}$ for $\Delta \varepsilon=0.6$ \\
FE average heating with loss tan $5 \times 10^{-4}$ & $0.9 \mathrm{~K}$ for $\Delta \varepsilon=2.7$ \\
FE pulse heating with loss tan $2 \times 10^{-3}$ & $\sim 0.4 \mathrm{~K}$ for $\Delta \varepsilon=0.6$ \\
FE average heating with loss tan $2 \times 10^{-3}$ & $\sim 3.5 \mathrm{~K}$ for $\Delta \varepsilon=2.7$ \\
\hline \hline
\end{tabular}

scaled tuner model may suffer a transmission loss no better than $\sim 0.7 \mathrm{~dB}$. In actuality, the measured transmission is worse. The best value obtains only when one uses either freshly applied liquid indium-gallium or solders the bars to the waveguide walls using indium. However, we were not able to apply more than $4 \mathrm{kV}$ to the soldered configurations; hence, we discuss below only the structures assembled with liquid indium-gallium. It is found that in the transmission drops when the voltage increases (see Fig. 8).

It is highly questionable, but still anticipated, that a development of coating layers, which do not degrade during brazing, and successful brazing of the ferroelectric and matching dielectric bars will eliminate losses beyond those in the bulk ceramics and metallic walls, as well as to lead to transmission being independent of applied voltage. As of now, several brazing attempts have revealed that the gold coating of the bar surfaces suffers badly when subjected to rapidly rising temperature and, in addition, the brazing atmosphere must be thoroughly controlled to avoid traces of oxygen.
Results of measurements of phase shift are presented in Fig. 9. Again, tests were made with gold-plated ferroelectric bars and matching slabs and the contact to copper walls was provided by liquid indium-gallium alloy. The obtained values of the phase shift are seen to be in good agreement with expectations. However, it seems that the coated ferroelectric samples suffer some sort of a "shock" when exposed to high voltages; as the result, the observed dielectric constant would not return to its original value once the voltage is completely removed. It is highly questionable if this effect is of hysteresis-like nature, that is, if the dielectric constant will return to its original value when the FE ceramic is biased with some voltage of the opposite sign. The shock nature of this effect suggests that some residual shift in the dielectric constant is going to either remain and only disappear with passing time, or even grow further when an increased bias (of the same or opposite sign) is being continuously applied. Further research is planned to address this issue; in particular, one should understand if the observed effects are due to the particular composition of the ceramic itself, or because of complex

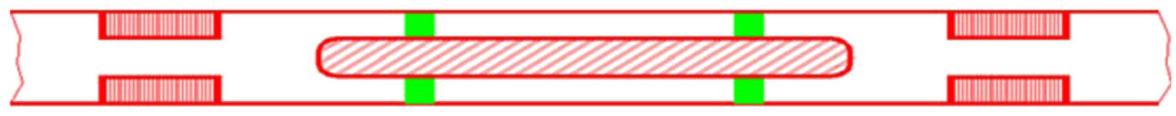

(a)

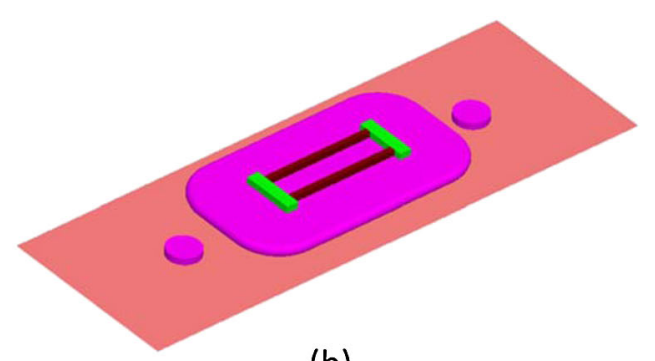

(b)

FIG. 7. (a) A cross-sectional side view of the one-third structure used for tests. Four rectangular boxes (two on the left, and two on the right) represent matching metal insertions; four square boxes (green) located above and below the electrode in the middle represent matching ceramic slabs. Ferroelectric rods are not seen in this cross section. (b) 3D view of the device used in the described below tests; the top wall (with two matching metal "tablets") and side walls are removed for clarity. 


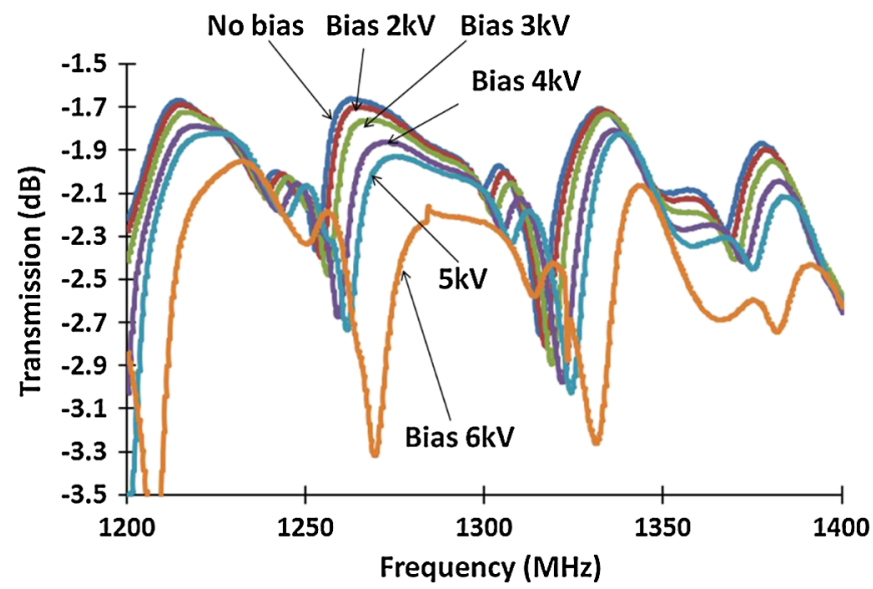

FIG. 8. The transmission drops when the voltage grows (see the magnitude change at $1.3 \mathrm{GHz}$ ).

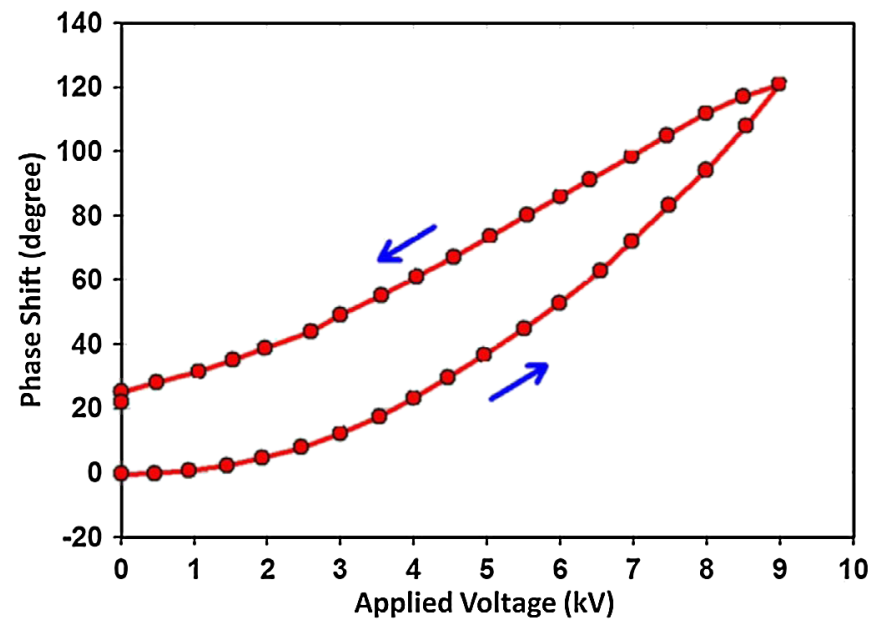

FIG. 9. Measured phase shift of rf signal transmitted through one-third section vs applied bias voltage.

physics happening in the transition layer between the ceramic and its metallization.

A vital property of any tuner is its response time, which for many accelerator applications should be less than 100 ns. Measurements of response time were made using the arrangement shown in Fig. 10. The high-voltage rise/ fall times from the available pulse generator were in the range of $\sim 100 \mathrm{~ns}$ (measured as the time difference from $5 \%$ to $95 \%$ of the voltage maximum). Switching speed measurements (each averaged over 16 shots) were processed by subtracting data with rf off from data taken with rf on, and are shown in Fig. 11.

The difference signal of $67 \mathrm{mV}$ from the mixer corresponds to a phase change of $77^{\circ}$. From these data, where the response time of the phase shifter is dominated by the 90 ns rise time of the voltage pulse, one infers that the response time to a step function voltage would be equal to or less than the delay time, namely, approximately $30 \mathrm{~ns}$. This could be interpreted to correspond to an average

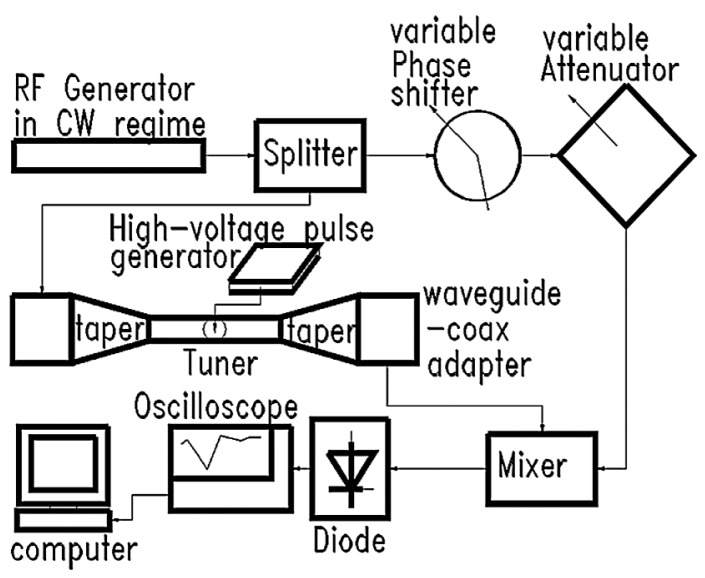

FIG. 10. The signal from the rf generator at $1290 \mathrm{MHz}$ is split in two. One portion is directed through a phase shifter and attenuator directly to a mixer, while the second portion is fed through the tuner input port, passes through the tuner, picked up at the tuner output port, and then is fed to the mixer. The resulting signal from the mixer is detected by a diode and monitored at an oscilloscope, and also captured by a computer for further signal processing [mainly via fast Fourier transformation (FFT)].

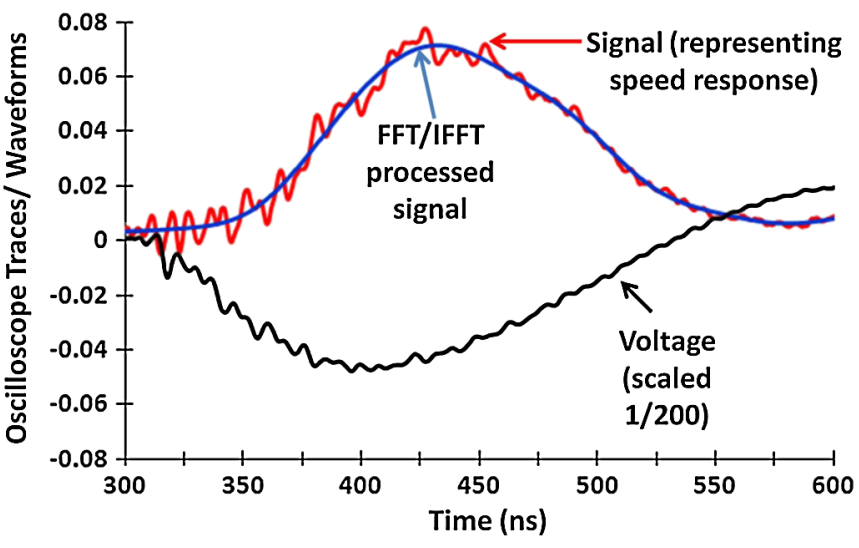

FIG. 11. Time response of phase shifter. Convex curve with wiggles (red) is the signal which is the difference between data with rf off and rf on. Smooth convex curve (blue) is the result of FFT/IFFT processing. The concave black curve is the highvoltage pulse with its peak value of $\sim 9.7 \mathrm{kV}$. It is seen that the time delay between the peak voltage and the peak variation in phase is $28 \mathrm{~ns}$. This value excludes delays in cables. The difference signal of $67 \mathrm{mV}$ from the mixer corresponds to a phase change of $77^{\circ}$. (One horizontal division is $50 \mathrm{~ns}$.)

switching rate of less than $0.5 \mathrm{~ns}$ for each degree of $\mathrm{rf}$ phase.

\section{OTHER DESIGNS}

As it has been mentioned, we considered several other designs for the phase shifter. Because of the nature of the article we shall describe them briefly here. 
Figure 12 presents the coaxial design. The ring (\#5) is ferroelectric material that is preceded by the matching ceramic (\#4). The gap is inevitable between the central part and the outer shell to apply the bias voltage. Consequently, one must employ a choke cavity (\#7). It is somewhat bulky design $(\varnothing 23 \mathrm{~cm} \times 56 \mathrm{~cm}$ height). It was found that the complexities and difficulties are hard to overcome when brazing both sides of the ferroelectric and/or insulation ceramic. Because of this, after a period of development we abandoned this version.

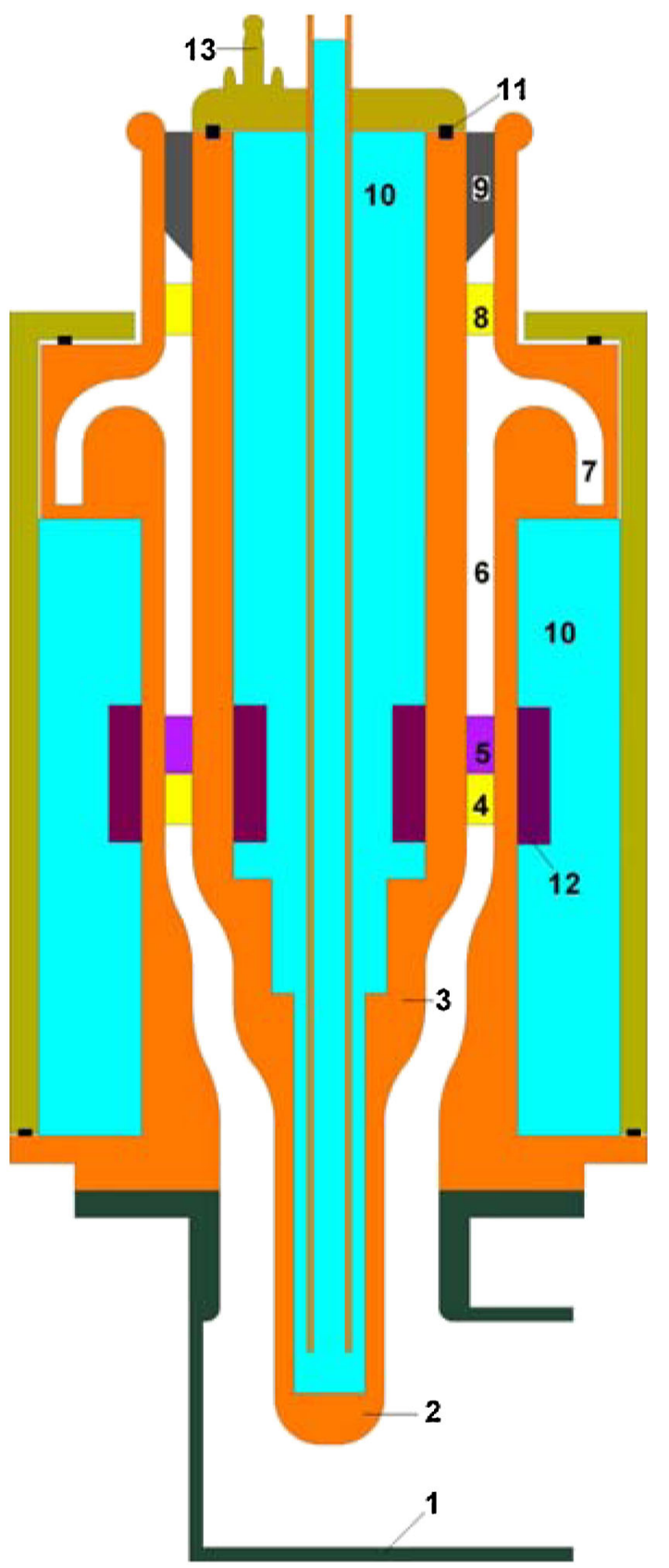

FIG. 12. Coaxial version (it is about $55 \mathrm{~cm}$ tall/ long, and $23 \mathrm{~cm}$ in diameter).
Figure 13 presents the planar-coax design. As before there is a gap between the cavity halves, and one must use a choke cavity. The ferroelectric ceramic (brown ring) is preceded by the matching ceramic (shown as yellow ring). The design is compact: $\varnothing 23 \mathrm{~cm} \times 12 \mathrm{~cm}$ height. Simulations of the full geometry show that the solution has many resonances in ferroelectric and matching ceramics. Unfortunately so far, we have failed to find a working solution for planar-coax geometry. However, the design is very attractive because of its simplicity. One may continue in two directions: (1) find a geometry modification in which the parasitic mode's frequencies are far from the working mode (if possible), and (2) carefully design the

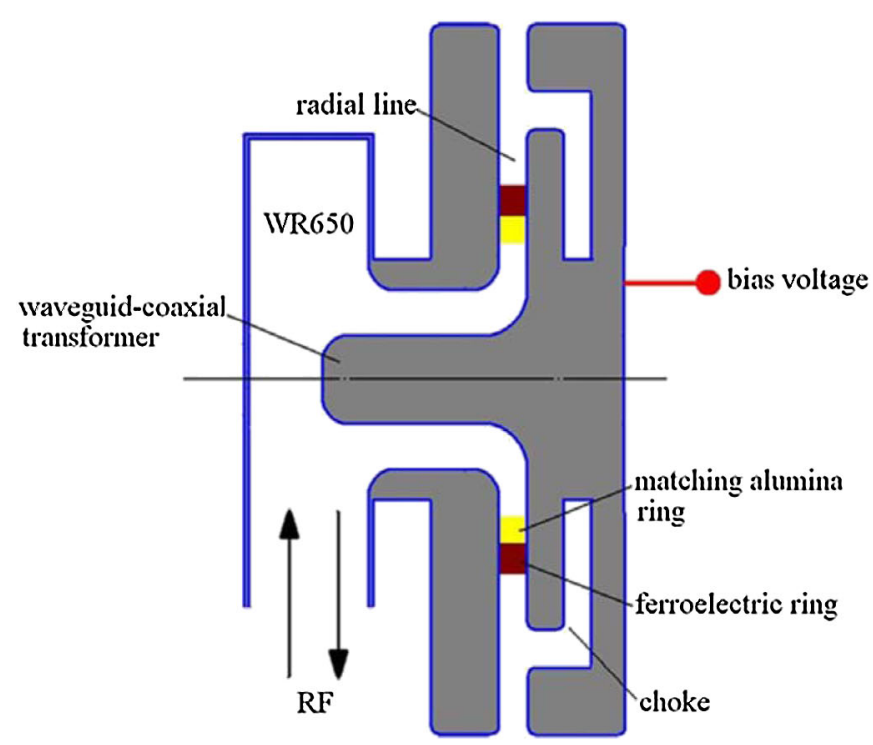

(a)

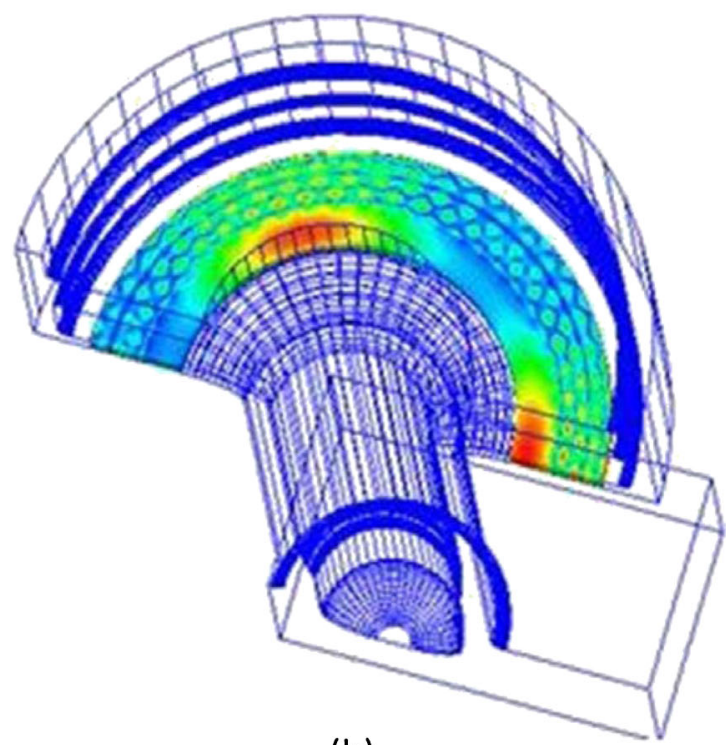

(b)

FIG. 13. (a) Planar-coax version (it is about $22 \mathrm{~cm}$ in diameter and $12 \mathrm{~cm}$ wide); and (b) example of a parasitic mode in the planar-coax version. 
coax-waveguide adapter in order not to excite parasitic modes. We should note that one of our co-authors has obtained a further monetary support to proceed in the aforementioned directions.

\section{CONCLUSIONS AND PLANS}

An rf-wave phase shifter based on a novel BST FE ceramic has been shown capable of delivering rapid phase switching (perhaps $<100 \mathrm{~ns}$ for shifts of $\sim 180$ degrees). That makes it an attractive candidate to externally tune the superconducting radiofrequency cavities for ERLs to reduce (at least by an order of magnitude) the rf-power requirements that arise because of phase instabilities of different origin, including microphonics as one of many.

The conducted research has revealed that several material issues must be addressed, including (a) redesigning the coating metallization layers; (b) brazing; (c) provisions to limit breakdowns at high bias voltages; and (d) redesigning the FE ceramic for L-band with low losses.

In a view of these issues, no further tests could be carried out, in particular until new improved FE ceramic samples with coating layers suitable for brazing would be delivered, but the schedule for this is uncertain.

The planned work also includes: (1) further developing the design for planar-coax geometry because it promises simplicity (relative to other designs) and thus low cost; and (2) proceeding with high-power tests but not necessary with the presented above $1 / 3$ model of the shifter. These efforts are underway.

Lastly, we note that the tuner in the configuration as described above was connected to a $1.3 \mathrm{GHz}$ cavity that is a mock-up of the superconducting rf-gun cavity designed to be used in the electron cooling project at BNL, see [11], and confirmed the capability of tuning of its resonance frequency [20].

\section{ACKNOWLEDGMENTS}

This work was supported by the Office of High Energy Physics, U.S. Department of Energy. We also acknowledge the help of Timergali Khabiboulline (FNAL), Harald Hahn (BNL), and E. M. Choi (formerly of BNL).

[1] S. Simrock et al., in Proceedings of the 20th Particle Accelerator Conference, Portland, OR, 2003 (IEEE, New York, 2003), p. 470.

[2] M. Liepe and S. Belomestnykh, in Proceedings of the 20th Particle Accelerator Conference, Portland, OR, 2003 (Ref. [1]), pp. 1326-1328.

[3] Y. Kang et al., in Proceedings of the 21st International Linac Conference, Gyeongju, Korea, 2002 (Pohang Accelerator Laboratory, Pohang, Korea, 2002), pp. 733-735.

[4] T. Zwart et al., in Proceedings of the 26th International Free Electron Laser Conference and 11th FEL User
Workshop, FEL 2004, Trieste, Italy, edited by René Bakker, Luca Giannessi, Marino Marsi, and Richard Walker (Comitato Conferenze Elettra, Trieste, Italy, 2004), pp. 542-545 [http://www.elettra.trieste.it/fel2004/ proceedings/].

[5] G. K. Davis and J. R. Delayen, in Proceedings of the 20th Particle Accelerator Conference, Portland, OR, 2003 (Ref. [1]), p. 1383.

[6] O. Kugeler, at the ERL09: Energy Recovery Linac, 45th ICFA Beam Dynamics Workshop, 2009, Cornell University [http://edms.classe.cornell.edu/agenda/ contributionDisplay.py?contribId=40\&amp; sessionId= 2\&amp; confId=7].

[7] D. Valuch et al., in Proceedings of the 9th European Particle Accelerator Conference, Lucerne, 2004 (EPSAG, Lucerne, 2004), pp. 959-961.

[8] D. Horan et al., in Proceedings of the 20th Particle Accelerator Conference, Portland, OR, 2003 (Ref. [1]), pp. 1177-1179.

[9] B. Foster et al., in Proceedings of the 21st Particle Accelerator Conference, Knoxville, 2005 (IEEE, Piscataway, NJ, 2005), p. 3123-3125.

[10] V. P. Yakovlev et al., in Proceedings of the 10th European Particle Accelerator Conference, Edinburgh, Scotland, 2006 (EPS-AG, Edinburgh, Scotland, 2006), pp. 487-489.

[11] I. Ben-Zvi, in Proceedings of the 10th European Particle Accelerator Conference, Edinburgh, Scotland, 2006 (Ref. [10]), pp. 940-944; See also R. Calaga, "SRF Cavities for High Current ERLs," BNL, 2006.

[12] O. A. Nezhevenko and V. P. Yakovlev, IEEE Transactions On Plasma Science, 2000, Vol. 28, No. 3 (IEEE Nuclear and Plasma Sciences Society, 2000), pp. 542-549 [http:// ieexplore.ieee.org/servlet/opac?punumber=27].

[13] D. Wang et al., in Proceedings of the 20th Particle Accelerator Conference, Portland, OR, 2003 (Ref. [1]), pp. 1300-1302.

[14] M. Liepe, at the ERL09: Energy Recovery Linac, 45th ICFA Beam Dynamics Workshop, 2009, Cornell University [http://edms.classe.cornell.edu/agenda/ contributionDisplay.py?contribId=37\&amp;sessionId= 2\&amp; confId=7].

[15] L.C. Sengupta, International Microwave Symposium, Boston, Massachusetts, 2000 [http://my.ece.ucsb.edu/ yorklab/Projects/Ferroelectrics/IMS2000\%20Workshop/ WFE002.pdf].

[16] D. Webb, International Microwave Symposium, Boston, Massachusetts, 2000 [http://my.ece.ucsb.edu/yorklab/ projects/Ferroelectrics/IMS2000\%20Workshop/ WFE004.pdf].

[17] A. B. Kozyrev et al., Tech. Phys. Lett. 24, 755 (1998).

[18] A. Kanareykin et al., in Proceedings of the 10th European Particle Accelerator Conference, Edinburgh, Scotland, 2006 (Ref. [10]), pp. 3251-3253.

[19] S. Yu. Kazakov et al., in Proceedings of the 2007 Particle Accelerator Conference, Albuquerque, New Mexico (IEEE, Albuquerque, New Mexico, 2007), pp. 596-598.

[20] H. Hahn et al., in Proceedings of the 23rd Particle Accelerator Conference, Vancouver, Canada, 2009 (IEEE, Piscataway, NJ, 2009) [http://trshare.triumf.ca/ $\sim$ pac09proc/Proceedings/papers/tu5pfp035.pdf]. 